\title{
Habitual levels of higher, but not medium or low, impact physical activity are positively related to lower limb bone strength in older women: findings from a population-based study using accelerometers to classify impact magnitude
}

\author{
K. Hannam ${ }^{1}$ - K. C. Deere ${ }^{1}$ A. Hartley ${ }^{1}$ U. A. Al-Sari ${ }^{1}$ - E. M. Clark ${ }^{1}$ - W. D. Fraser $^{2}$ \\ J. H. Tobias ${ }^{1}$
}

Received: 22 September 2016/Accepted: 29 November 2016/Published online: 13 December 2016

(C) The Author(s) 2017. This article is an open access publication

\begin{abstract}
Summary This study assessed the effect of accelerometrymeasured higher impacts resulting from habitual weightbearing activity on lower limb bone strength in older women. Despite higher impacts being experienced rarely in this population-based cohort, positive associations were observed between higher vertical impacts and lower limb bone size and strength.

Introduction We investigated whether the benefit of habitual weight-bearing physical activity (PA) for lower limb bone strength in older women is explained by exposure to higher impacts, as previously suggested by observations in younger individuals.

Methods Four hundred and eight women from the Cohort for Skeletal Health in Bristol and Avon (COSHIBA), mean 76.8 years, wore tri-axial accelerometers at the waist for a mean of 5.4 days. $Y$-axis peaks were categorised, using previously identified cutoffs, as low $(0.5-1.0 \mathrm{~g})$, medium (1.0 $1.5 \mathrm{~g})$, and higher $(\geq 1.5 \mathrm{~g})$ impacts. Mid and distal peripheral quantitative computed tomography scans of the tibia and radius were performed, as were hip and lumbar spine Dual Xray Absorptiometry (DXA) scans. Regressions between (log transformed) number of low, medium and high impacts, and
\end{abstract}

Electronic supplementary material The online version of this article (doi:10.1007/s00198-016-3863-5) contains supplementary material, which is available to authorized users.

J. H. Tobias

Jon.Tobias@bristol.ac.uk

1 Musculoskeletal Research Unit, University of Bristol School of Clinical Sciences, Southmead Hospital, Bristol BS10 5NB, UK

2 University of East Anglia, Norwich, UK bone outcomes were adjusted for artefact error grade, age, height, fat and lean mass and impacts in other bands.

Results Eight thousand eight hundred and nine $(4047,16,882)$ low impacts were observed during the measurement week, $345(99,764)$ medium impacts and $42(17,106)$ higher impacts (median with 25th and 75th quartiles). Higher vertical impacts were positively associated with lower limb bone strength as reflected by cross-sectional moment of inertia (CSMI) of the tibia $[0.042(0.012,0.072) p=0.01]$ and hip $[0.067(0.001,0.133) p=0.045]$ (beta coefficients show standard deviations change per doubling in impacts, with $95 \%$ confidence interval). Higher impacts were positively associated with tibial periosteal circumference (PC) $[0.015(0.003$, 0.027) $p=0.02]$, but unrelated to hip BMD. Equivalent positive associations were not seen for low or medium impacts. Conclusions Despite their rarity, habitual levels of higher impacts were positively associated with lower limb bone size and strength, whereas equivalent relationships were not seen for low or medium impacts.

Keywords G-force · Hip BMD · Tibial pQCT

\section{Introduction}

Physical activity (PA) is thought to have a positive effect on bone health throughout the life course, through maximising peak bone mass in childhood and adolescence and minimising age-related bone loss in older adults. Research efforts are now increasingly focussed on optimising the non-pharmacological benefits of activity by determining optimal type, magnitude and duration of activity to maximise and maintain bone strength to ultimately reduce fracture risk. Interventional studies suggest that weight-bearing activities producing high 
vertical impacts improve lower limb bone strength in children and adolescents [1], pre-[2] and post-menopausal women [3] and older men [4]. The suggestion that the skeleton is primarily influenced by high impacts is consistent with laboratory studies indicating a dose response between strain level and bone formation [5]. Suppression of bone turnover may also contribute to the positive effects of mechanical bone loading, as exemplified by findings that $\mathrm{C}$-terminal telopeptide $(\beta$ CTX) was reduced following an exercise intervention in older obese adults [6], and $\beta$-CTX levels in athletes decrease following exercise [7], although other studies show conflicting findings [8].

Observational studies also suggest that the amount of dayto-day participation in high-impact PA is positively related to lower limb bone strength, based on PA questionnaires in which different activities are graded according to impact level $[9,10]$. Accelerometers provide more objective measures of PA exposure. However, in generating counts per minutes, widely used actigraph accelerometers use proprietary software which combines movement frequency and intensity, rather than evaluating peak acceleration magnitude per se. To define PA in terms of impacts to which the skeleton responds, the Newtest device was developed to measure the number of acceleration peaks according to $g$ level. This starts sampling vertical accelerations each time these exceed a threshold of $0.3 \mathrm{~g}$, following which the maximum acceleration value is identified and recorded, representing the acceleration peak for a given movement [11]. Using this device, the benefits of weight-bearing PA for hip BMD in premenopausal women were found to be explained by impacts beyond $3.9 \mathrm{~g}$ [12]. Similarly, in adolescents from the Avon Longitudinal Study of Parents and Children (ALSPAC), relationships between PA and hip BMD and tibial geometry were solely explained by vertical impacts beyond $4.2 \mathrm{~g}$, despite their rarity (e.g. median of 7.8 per day in females) $[13,14]$. However, to what extent equivalent relationships are observed in older populations are currently unknown.

Improvements in technology have since made it possible to store 7 days of continuous accelerometer recordings, enabling acceleration peaks to be identified retrospectively by applying data processing algorithms to raw data [15]. In addition, if acceleration peaks are recorded in time sequence, artefacts and errors can be identified more readily [15]. Applying this method to a population-based sample of older individuals, we recently found that virtually no acceleration peaks occur beyond $2 \mathrm{~g}$, reflecting their lack of participation in highimpact PA. Therefore, although a $1.5 \mathrm{~g}$ threshold can be applied in this group to define relatively high vertical impacts, application of the conventional 4-g osteogenic threshold does not appear to be feasible. That said, osteogenic thresholds as defined in younger populations may not be applicable to older individuals, in whom lower levels of impacts may produce equivalent strains to those resulting from higher impacts in younger people, due to a weaker skeleton.

In the present study, we investigated whether skeletal benefits of habitual weight-bearing PA can be explained in terms of exposure to vertical impacts $>1.5 \mathrm{~g}$ in a population-based cohort of older women, despite the rarity of these impacts and the relatively low threshold used to define higher impacts. Specifically, we examined (i) whether habitual exposure to higher, but not medium or low, vertical impacts as defined in this way are positively related to lower limb bone strength, (ii) whether relationships between higher impacts and lower limb bone strength are explained by changes in geometry and/or $\mathrm{BMD}$, and (iii) whether alterations in bone formation or resorption underlie these associations as reflected by measurement of N-terminal propeptide of type I collagen (P1NP) and $\beta$-CTX, respectively.

\section{Materials and methods}

\section{Study design}

The VIBE study included participants from the Cohort for Skeletal Health in Bristol and Avon (COSHIBA) on which the present analysis is based. COSHIBA is a populationbased cohort of 3200 women who were originally recruited in the Bristol and Avon area during 2007-2009 from GP surgeries with the sole-entry criteria of date of birth falling between 1 January 1927 and 31 December 1942 [16, 17]. Of the original 3200 COSHIBA participants who remained resident in the Bristol and Avon area, 1286 consented to be contacted about future research studies in 2014 and were eligible for inclusion in the VIBE study. Eligible participants were subsequently invited in alphabetical order, stopping after 1064 by which time available clinic assessment slots had been filled (see Fig. 1). COSHIBA participants attended VIBE clinic sessions throughout 2015 during which measures of skeletal health were obtained, immediately following which the 7day accelerometry data collection was commenced. An explanation of the assessments followed by written informed consent and a verbal consent check before each procedure was obtained from every participant. The study was approved by the National Research Ethics Committee (14/SW/0138) in Bristol (South West-Frenchay).

\section{Accelerometry}

Participants who consented to 7-day PA monitoring were provided with a GCDC X15-1c triaxial accelerometer (Gulf Coast Data Concepts, Waveland, Mississippi) along with a size-specific elasticated belt, a daily time log (recording time accelerometer was taken on/off and whether the day was 


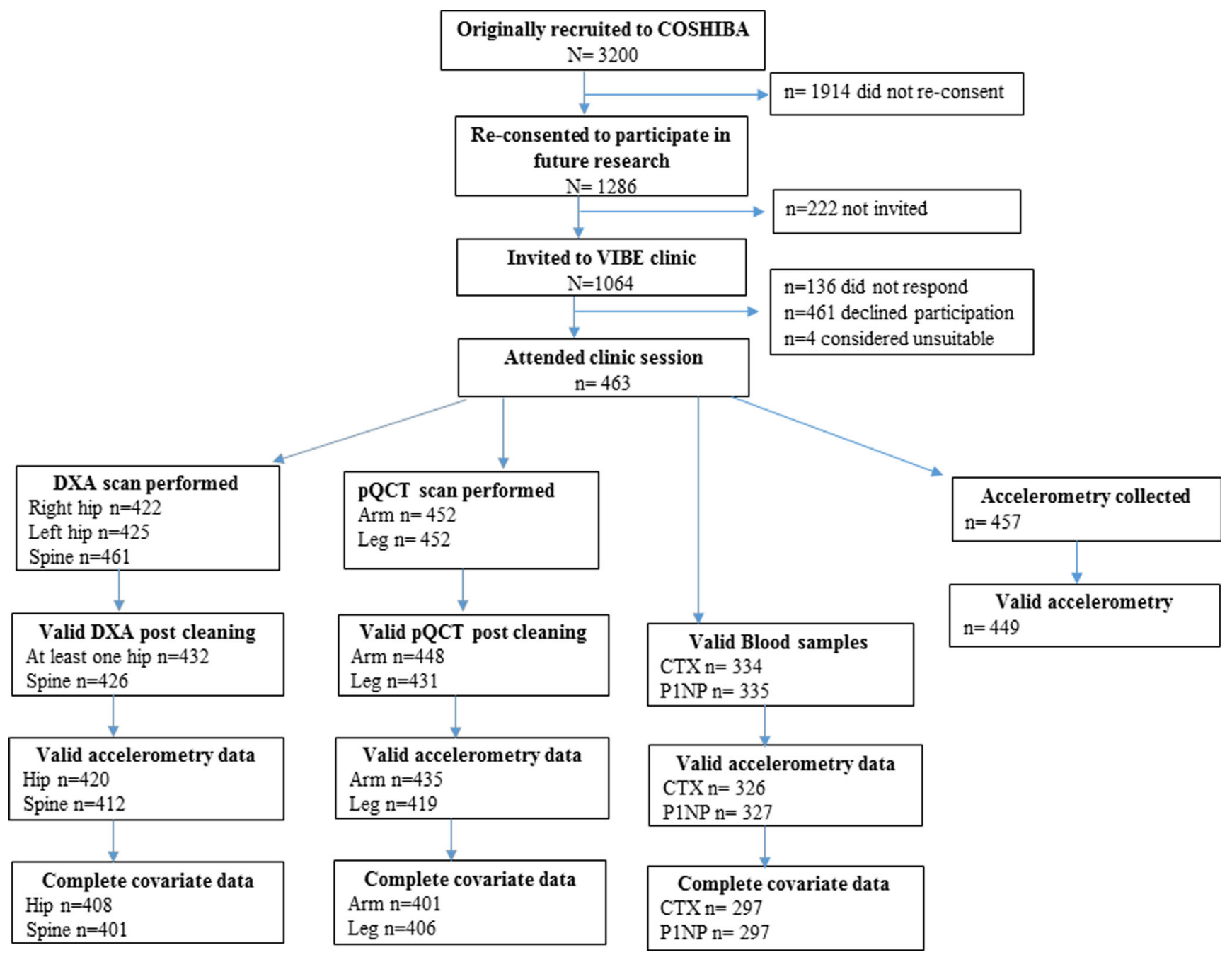

Fig. 1 Flow diagram showing the recruitment of participants to the study

normal in terms of activity) and a stamped addressed package along with written and verbal instructions. Participants were instructed to wear the accelerometer securely positioned over their right hip pointing toward the centre of their body for 7 consecutive days, only to be removed for sleeping, washing and swimming. Accelerometers were configured by the research team prior to participant use with a sampling frequency of $50 \mathrm{~Hz}$. Raw accelerometry data were imported to Stata 13 (StataCorp, College Station, TX) for standardised processing using custom designed code (explained in detail elsewhere [15]). Briefly, accelerometry data were cleaned to remove movement artefacts and non-wear time. A given day was excluded if less than $10 \mathrm{~h}$ of valid recording time was obtained, to ensure that each day's recording was representative of the total amount of PA undertaken [15]. Sensitivity analyses were performed with the restriction of only those with $\geq 3$ valid days, as previously applied [15]. As previously described, $Y$ axis peaks were identified based on accelerations that were higher than the preceding and subsequent readings and grouped into three bands to reflect low $(\geq 0.5$ to $<1.0 \mathrm{~g}$ ), medium $(\geq 1.0$ to $<1.5 \mathrm{~g})$ and high $(\geq 1.5 \mathrm{~g})$ impact (movement $\leq 0.5$ g were deemed sedentary activity and excluded) over and above $1 \mathrm{~g}$ of earth's gravitational force [15]. Activity data were normalised based on 7 valid days $(\geq 10$-h recording time) of $14 \mathrm{~h}$.

\section{pQCT}

All COSHIBA participants who attended the outcome assessment clinic were offered a peripheral quantitative computed tomography (pQCT) at the tibia and radius using the Stratec XCT2000L (StraTec Medizintechnik, Pforzheim, Germany), which was performed as per standard operating procedures. The right arm $(4$ and $60 \%$ of the radius) and leg (4, 50 and $66 \%$ of the tibia) were scanned unless there was a history of recent fracture, or excessive tremor or metal pins and plates were present. Measurements were obtained for cortical BMD $\left(\mathrm{BMD}_{\mathrm{c}}\right.$, $\left.\mathrm{mg} / \mathrm{cm}^{3}\right)$ and trabecular BMD $\left(\mathrm{BMD}_{\mathrm{t}}, \mathrm{mg} / \mathrm{cm}^{3}\right)$ using $\mathrm{XCT}$ custom software. Additional pQCT variables were 
derived from a circular ring model including cortical thickness $(\mathrm{CT}, \mathrm{mm})$; periosteal circumference $(\mathrm{PC}, \mathrm{mm})$; cross-sectional moment of inertia $\left(\mathrm{CSMI}, \mathrm{mm}^{4}\right)$ and strength strain index $\left(\mathrm{SSI}, \mathrm{mm}^{3}\right)$, using a threshold of $350 \mathrm{mg} / \mathrm{cm}^{3}$ to define bone. Trabecular BMD measurements were taken from the $4 \%$ site of the tibia and radius and all other measurements were taken or derived from results at the $60 \%$ site for the radius and $50 \%$ site for the tibia. All scan images were visually checked at the time the scan was performed and assigned a grade $(0-5)$ based on the extent of artefact present. A score of 0 indicated no artefact, minimal artefact (score of 1), small (2), moderate (3), large - no analysis possible but features are still recognisable (4), and large-recognition of anatomical features not possible (5). The clinic workers were advised to repeat the scan if appropriate where a grade 3-5 was assigned. Analyses were based on scans with artefact scores of $0-3$, of which 96.5 and $80.2 \%$ were graded $0-2$ for tibial and radial scans, respectively. Repeat tibial pQCT scans were performed in 20 COSHIBA participants within 1 month of the original scan. Within subject coefficients of variation at the $50 \%$ site were $2.46 \%$ for PC, $0.93 \%$ for $\mathrm{BMD}_{\mathrm{c}}, 2.97 \%$ for $\mathrm{CT}, 8.27 \%$ for CSMI, $5.49 \%$ for SSI and $5.87 \%$ for $\mathrm{BMD}_{\mathrm{t}}$.

\section{Anthropometric and DXA variables}

Dual x-ray absorptiometry scan (DXA) was performed on a GE Healthcare Lunar Prodigy. Standing height was measured to the nearest millimetre using a Harpenden stadiometer (Holtain Ltd., Crymych, UK) and weight was measured to the nearest $50 \mathrm{~g}$ using Tanita weighing scales (Tanita UK Ltd., Uxbridge, UK). Consenting participants who were able to transfer onto the DXA scan bed unaided underwent a total body (generating fat and lean mass (FM and LM, kg), lumbar spine (L1-L4 (LS) BMD, g/ $\mathrm{cm}^{2}$ ) and left and right hip scans (generating total hip $(\mathrm{TH})$ and femoral neck (FN) BMD, $\mathrm{g} / \mathrm{cm}^{2}$ ). The manufacturer's automated advanced hip analysis (AHA) software was used to derive minimum neck width (MNW, mm) and cross-sectional moment of inertia (CSMI, $\mathrm{mm}^{4}$ ). For the purpose of analyses, the right hip results were used unless of joint replacement, recent fracture or significant artefact. DXA scans were cleaned using a systematic approach to correct for positioning errors, where possible, and identification and coding of minor and major artefacts. A substantial proportion of lumbar scans were found to have significant lumbar spondylosis, necessitating the exclusion of one or more lumbar vertebrae, which was performed in preference to excluding the whole lumbar spine scan. Repeat total body and hip DXA scans were performed in 20 COSHIBA participants within 1 month of the original scan. Within subject coefficients of variation were $1.65 \%$ for FM, $1.48 \%$ for
LM, $1.51 \%$ for TH BMD, $1.86 \%$ for FN, $1.49 \%$ for MNW and $5.96 \%$ for CSMI.

\section{Bone turnover markers}

Participants were eligible to provide a blood sample at the clinic session provided they gave informed consent, had not been diagnosed with a bleeding disorder, were not currently taking anti-coagulant drugs and had not been recently diagnosed with anaemia, hepatitis $\mathrm{B}$ and $\mathrm{C}$ or HIV/AIDS positive. Participants were requested to fast for at least $4 \mathrm{~h}$ prior to their appointment and last time since eating/drinking was recorded. All samples were centrifuged at the study centre laboratory as soon as possible after obtaining the sample $(\leq 20 \mathrm{~min})$ and labelled aliquots were stored at $-80^{\circ} \mathrm{C}$. Samples were then sent for analyses of $\beta$-CTX and propeptide of type I collagen (P1NP) at the Bioanalytical facility at the University of East Anglia. Electrochemiluminescence immunoassays (ECLIA) on a Cobas 1600i analyser (Roche Diagnostics, Lewes, UK) were used, with detection limits of $0.01 \mu \mathrm{g} / \mathrm{L}(\beta-\mathrm{CTX})$ and $8 \mu \mathrm{g} / \mathrm{L}$. (P1NP); inter and intra-assay coefficients of variation $(\mathrm{CVs})$ were $<4.0 \%$ across the working range of both assays.

\section{Additional confounders}

On attending the research clinic, participants were also asked to complete a questionnaire asking about potential confounders which were also analysed as part of the study. These included self-reported health status (5-point scale from very poor to very good), co-morbidities potentially influencing PA participation (i.e. respiratory disease, cardiovascular disease, arterial disease, stroke, Parkinson's disease and arthritis), which were then summed, and socioeconomic status based on their main occupation (and that of their spouse if married) during their working life. Each occupation was assigned a SOC90 (Standard Occupation Classification) code and collapsed to nine groups, the highest SOC90 code between the participant and spouse being assigned where both occupations were provided. Whether subjects were currently taking bone active agents (i.e. bisphosphonates, denosumab or strontium) was also recorded, as was the extent of PA participation below 18 years of age, and between age 18-29.

\section{Statistical analyses}

Descriptive data on participant characteristics, accelerometry, DXA, pQCT and bone turnover markers were expressed as means and medians with standard deviations (SD) and the 25th and 75 th percentiles. Accelerometry data were positively skewed and thus log transformed for regression analyses, which led to normalisation of data as assessed by visual inspection of histograms. Multivariate linear regression analyses were performed to explore associations between the number of $Y$-axis peaks, and 
DXA, pQCT and bone turnover markers, in the study population as a whole. A three-band model was used whereby $0.5<g \leq 1.0$, $1.0<g \leq 1.5$ and $g>1.5$ counts were entered simultaneously, after checking model parameters $\left(r^{2}\right.$ and Akaike information criterion (AIC)). All outcome variables were standardised and results presented indicate a SD change in the outcome variable per doubling in activity. Three models were used to explore associations between activity and bone outcome measures, a minimally adjusted model including only age as a potential confounder, a second model including age, height, fat and lean mass, and a third model incorporating additional adjustment for impacts in other bands. Analyses of radial and tibial pQCT data also included adjustment for error grading at the relevant site in both models, and those for bone turnover markers whether samples were am/pm. Further models were included in the analyses of tibial pQCT adjusting for $\beta$-CTX, P1NP, socioeconomic status and health status as assessed by questionnaire.

\section{Results}

\section{Subject characteristics}

1064 COSHIBA participants were invited to the VIBE research clinic of whom 463 attended a clinic session and 449 had valid accelerometry data. Complete datasets ranged from 408 (hip DXA), 406 (tibial pQCT) and 297 (bone turnover markers) (see Fig. 1). Based on individuals with complete hip DXA results, women were a mean age of 76.7 years, and wore accelerometers for a mean of 5.4 days (Table 1). A median of 8809, 345 and 42 low, medium and higher impacts per week were observed, respectively. COSHIBA participants who were invited to participate but did not attend a clinic session were a mean of 80.7 years of age.

\section{Impacts versus pQCT parameters}

Linear regression analyses were performed to examine, in turn, the associations between the number of low, medium and high vertical impacts recorded for each individual and bone measures. Higher impacts were positively associated with tibial PC, CSMI and SSI in all models, with strongest associations observed in our fully adjusted model (model 3 ) [tibial PC 0.015 (0.003, 0.027); CSMI 0.042 (0.012, 0.072); SSI $0.034(0.009,0.058)$ (Table 2). Low and medium impacts were unrelated to these parameters.

High, medium and low impacts were inversely related to $\mathrm{BMD}_{\mathrm{c}}$ and $\mathrm{BMD}_{\mathrm{t}}$ in analyses adjusted for age only (model 1); however, these associations were largely attenuated by further adjustment for height and body composition (models 2 and 3). No associations were observed between impacts and CT in models 1 and 2. However, in model 3 (further adjustment for other bands), low and medium impacts showed inverse associations with CT. With the exception of a positive association between low-impact PA and radial $\mathrm{BMD}_{\mathrm{c}}$ in model 3, little relationship was observed between impacts and radial pQCT parameters (supplementary Table 1).

\section{Impacts versus DXA parameters}

Higher impacts were unrelated to CSMI in analyses models 1 or 2 (Table 3). However, higher impacts were positively associated with CSMI in model 3 [0.067 $(0.001,0.133)]$ (beta coefficient and $95 \%$ confidence interval). Higher impacts were unrelated to other hip geometric parameters, to LS BMD, and to TH and FN $\mathrm{BMD}$, in all models. Medium impacts were inversely related to CSMI in all models, to MNW in model 2 only, and to LS BMD only in model 1 . Low impacts showed a similar pattern in that they were inversely related to CSMI (models 1 and 2), MNW (model 2) and LS BMD (model 1). In addition, low impacts were inversely related to TH BMD $[-0.071(-0.134,-0.008)]$ and a lesser extent FN BMD $[-0.059(-0.122,0.003)]$.

\section{Impacts versus $\beta-C T X$ and P1NP}

Higher impacts were positively related to $\beta$-CTX in model 1 $[0.101(0.040,0.162)]$, which was slightly attenuated in model $2[0.088(0.026,0.150)$, and further attenuated in model $3[0.085$ $(-0.017,0.187)]$ (Table 4). Medium and low impacts showed a similar positive association with $\beta$-CTX compared to that seen high impacts, with progressive attenuation with further adjustment. Higher impacts were positively related to P1NP in all models with strongest associations observed in model $3[0.127$ $(0.031,0.224)]$. Weaker associations were observed between low and medium impacts and P1NP in models 1 and 2, whereas these associations were fully attenuated in model 3 .

\section{Sensitivity analyses and further confounder adjustment}

Similar results were obtained when analyses were restricted to participants with accelerometry data collected for a minimum of three valid days (results not shown). Further models were explored to analyse the role of different confounders, in 373 participants in whom additional information was available from questionnaires on socioeconomic status as reflected by SOC 90, comorbidities and concurrent use of bone-active medication (supplementary Table 2). Higher impacts showed similar relationships with tibial PC and CSMI to those seen in the wider cohort, with little change in beta coefficients following adjustment for any of the confounders examined (supplementary Table 3). We also examined the role of past weight-bearing PA, but no relationship was evident between levels of impacts $>1.5 \mathrm{~g}$ and extent of self-reported participation in weight-bearing PA $<18$ years, or 18-29 years, and results were unaffected by adjustment for past activity (results not shown). Finally, we examined whether associations between impacts and bone turnover 
Table 1 Descriptive statistics

\begin{tabular}{|c|c|c|c|c|}
\hline & Number & Median & $\mathrm{p} 25$ & $\mathrm{p} 75$ \\
\hline Age (years) & 408 & 76.2 & 74.6 & 78.1 \\
\hline Height (cm) & 408 & 159.1 & 154.6 & 162.8 \\
\hline Weight (kg) & 408 & 67.0 & 59.8 & 76.1 \\
\hline Accelerometer wear time (valid days) & 408 & 6.0 & 5.0 & 6.0 \\
\hline Low band peaks $(\geq 0.5$ to $<1.0 \mathrm{~g}$ ) & 408 & 8809 & 4047 & 16,882 \\
\hline Medium band peaks $(\geq 1.0$ to $<1.5 \mathrm{~g})$ & 408 & 345 & 99 & 764 \\
\hline High band peaks $\geq 1.5 \mathrm{~g}$ & 408 & 42 & 17 & 106 \\
\hline \multicolumn{5}{|l|}{ Tibial pQCT measures } \\
\hline $\mathrm{PC}(\mathrm{mm})$ & 406 & 69.8 & 67.4 & 73.2 \\
\hline CT (mm) & 406 & 4.8 & 4.2 & 5.4 \\
\hline $\mathrm{BMD}_{\mathrm{c}}\left(\mathrm{mg} / \mathrm{cm}^{3}\right)$ & 406 & 1121 & 1090 & 1146 \\
\hline $\mathrm{BMD}_{\mathrm{t}}\left(\mathrm{mg} / \mathrm{cm}^{3}\right)(4 \%)$ & 401 & 306 & 292 & 326 \\
\hline $\operatorname{CSMI}\left(\mathrm{mm}^{4}\right)$ & 406 & 10,567 & 9131 & 12,251 \\
\hline $\mathrm{SSI}\left(\mathrm{mm}^{3}\right)$ & 406 & 885 & 782 & 989 \\
\hline \multicolumn{5}{|l|}{ Radial pQCT measures } \\
\hline $\mathrm{PC}(\mathrm{mm})$ & 401 & 37.9 & 36.2 & 40.0 \\
\hline $\mathrm{CT}(\mathrm{mm})$ & 401 & 2.4 & 2.1 & 2.8 \\
\hline $\mathrm{BMD}_{\mathrm{c}}\left(\mathrm{mg} / \mathrm{cm}^{3}\right)$ & 401 & 1104 & 1070 & 1127 \\
\hline $\mathrm{BMD}_{\mathrm{t}}\left(\mathrm{mg} / \mathrm{cm}^{3}\right)(4 \%)$ & 423 & 332 & 322 & 343 \\
\hline $\mathrm{CSMI}\left(\mathrm{mm}^{4}\right)$ & 401 & 892 & 705 & 1107 \\
\hline $\mathrm{SSI}\left(\mathrm{mm}^{3}\right)$ & 401 & 134.8 & 113.2 & 162.4 \\
\hline \multicolumn{5}{|l|}{ DXA measures } \\
\hline FM (kg) & 408 & 27.2 & 22.2 & 32.9 \\
\hline LM (kg) & 408 & 37.6 & 34.6 & 41.0 \\
\hline TH BMD $\left(\mathrm{g} / \mathrm{cm}^{2}\right)$ & 408 & 0.86 & 0.78 & 0.97 \\
\hline $\mathrm{FN}(\mathrm{g} / \mathrm{cm} 2)$ & 408 & 0.82 & 0.74 & 0.92 \\
\hline MNW (mm) & 408 & 30.6 & 29.1 & 31.80 \\
\hline $\operatorname{CSMI}\left(\mathrm{cm}^{4}\right)$ & 408 & 9.1 & 7.6 & 10.5 \\
\hline LS BMD $\left(\mathrm{g} / \mathrm{cm}^{2}\right)$ & 401 & 1.0 & 0.89 & 1.2 \\
\hline \multicolumn{5}{|l|}{ Bone turnover markers } \\
\hline$\beta-\mathrm{CTX}(\mu \mathrm{g} / \mathrm{L})$ & 293 & 0.39 & 0.27 & 0.53 \\
\hline P1NP $(\mu \mathrm{g} / \mathrm{L})$ & 293 & 42.6 & 32.6 & 52.5 \\
\hline
\end{tabular}

Table shows participant characteristics including DXA measures [ $F M$ fat mass, $L M$ lean mass, $T H$ total hip, $F N$ femoral neck and $L S$ lumbar spine BMD, $M N W$ minimum femoral neck width and CSMI cross sectional moment of inertia ], pQCT [PC periosteal circumference, $C T$ cortical thickness $(\mathrm{CT}), B M D_{c}$ cortical BMD, $B M D_{t}$ trabecular density, $C S M I$ cross-sectional moment of inertia and SSI strength strain index], and bone turnover markers [ $\beta$ $C T X$ C-terminal telopeptide and $P I N P$ propeptide of type I collagen]

markers might explain those with tibial pQCT parameters. Higher impacts showed equivalent associations with tibial PC and CSMI in the subset of participants in whom bone turnover marker data were also available, as compared with the larger sample shown in Table 3; relationships were unaffected by additional adjustment for $\beta$-CTX or P1NP (supplementary Table 4).

\section{Discussion}

We examined the relationship between PA and bone health measures in a population-based cohort of older women, based on classification of vertical movements from accelerometers according to level of impact. Based on our fully adjusted model, we found that higher, but not medium or low, vertical impacts were positively related to estimated bone strength as reflected by hip and tibial CSMI, and tibial SSI. The associations we observed with lower limb strength appeared to largely reflect changes in overall bone size since higher impacts were also positively related to tibial $\mathrm{PC}$, whereas little relationship was evident with BMD. Taken together, our findings are consistent with the possibility that, despite their rarity and the relatively low $g$ threshold used to define them, habitual 
Table 2 Associations between vertical impacts and tibia $\mathrm{pQCT}$ measures

\begin{tabular}{|c|c|c|c|c|c|c|c|c|c|c|c|c|c|}
\hline \multirow[t]{2}{*}{ Tibia pQCT variable } & \multirow[t]{2}{*}{ Model } & \multicolumn{4}{|c|}{ Low impacts } & \multicolumn{4}{|c|}{ Medium impacts } & \multicolumn{4}{|c|}{ Higher impacts } \\
\hline & & Beta & Lower CI & Upper CI & $p$ & Beta & Lower CI & Upper CI & $p$ & Beta & Lower CI & Upper CI & $p$ \\
\hline \multirow[t]{3}{*}{$\mathrm{PC}(\mathrm{mm})$} & Model 1 & 0.000 & -0.011 & 0.011 & 0.991 & 0.003 & -0.005 & 0.011 & 0.485 & 0.010 & 0.001 & 0.018 & 0.022 \\
\hline & Model 2 & 0.002 & -0.009 & 0.013 & 0.686 & 0.004 & -0.004 & 0.011 & 0.347 & 0.009 & 0.001 & 0.016 & 0.022 \\
\hline & Model 3 & -0.002 & -0.021 & 0.018 & 0.866 & -0.007 & -0.024 & 0.010 & 0.436 & 0.015 & 0.003 & 0.027 & 0.017 \\
\hline \multirow[t]{3}{*}{$\mathrm{CT}(\mathrm{mm})$} & Model 1 & -0.014 & -0.039 & 0.011 & 0.264 & -0.014 & -0.032 & 0.004 & 0.128 & 0.001 & -0.018 & 0.020 & 0.936 \\
\hline & Model 2 & 0.011 & -0.016 & 0.038 & 0.412 & -0.004 & -0.023 & 0.014 & 0.629 & 0.002 & -0.016 & 0.021 & 0.794 \\
\hline & Model 3 & 0.058 & 0.010 & 0.105 & 0.018 & -0.053 & -0.095 & -0.011 & 0.013 & 0.022 & -0.008 & 0.051 & 0.145 \\
\hline \multirow[t]{3}{*}{$\mathrm{BMD}_{\mathrm{c}}\left(\mathrm{mg} / \mathrm{m}^{3}\right)$} & Model 1 & -0.014 & -0.029 & 0.000 & 0.050 & -0.010 & -0.021 & 0.000 & 0.055 & -0.011 & -0.022 & 0.000 & 0.057 \\
\hline & Model 2 & -0.008 & -0.024 & 0.008 & 0.330 & -0.007 & -0.018 & 0.004 & 0.205 & -0.009 & -0.021 & 0.002 & 0.095 \\
\hline & Model 3 & -0.001 & -0.030 & 0.028 & 0.926 & 0.001 & -0.025 & 0.027 & 0.925 & -0.010 & -0.028 & 0.008 & 0.281 \\
\hline \multirow[t]{3}{*}{$\mathrm{BMD}_{\mathrm{t}}\left(\mathrm{mg} / \mathrm{cm}^{3}\right)$} & Model 1 & -0.081 & -0.143 & -0.019 & 0.010 & -0.066 & -0.110 & -0.022 & 0.004 & -0.052 & -0.099 & -0.006 & 0.028 \\
\hline & Model 2 & -0.032 & -0.101 & 0.038 & 0.370 & -0.043 & -0.090 & 0.003 & 0.067 & -0.041 & -0.088 & 0.006 & 0.086 \\
\hline & Model 3 & 0.062 & -0.061 & 0.186 & 0.322 & -0.070 & -0.179 & 0.039 & 0.207 & -0.010 & -0.086 & 0.066 & 0.799 \\
\hline \multirow[t]{3}{*}{$\mathrm{CSMI}\left(\mathrm{mm}^{4}\right)$} & Model 1 & 0.000 & -0.029 & 0.028 & 0.984 & 0.006 & -0.015 & 0.026 & 0.595 & 0.026 & 0.005 & 0.048 & 0.017 \\
\hline & Model 2 & 0.013 & -0.014 & 0.040 & 0.345 & 0.010 & -0.008 & 0.029 & 0.282 & 0.024 & 0.005 & 0.042 & 0.012 \\
\hline & Model 3 & 0.016 & -0.033 & 0.064 & 0.524 & -0.031 & -0.073 & 0.012 & 0.159 & 0.042 & 0.012 & 0.072 & 0.006 \\
\hline \multirow[t]{3}{*}{$\mathrm{SSI}\left(\mathrm{mm}^{3}\right)$} & Model 1 & -0.008 & -0.032 & 0.016 & 0.495 & -0.001 & -0.019 & 0.016 & 0.884 & 0.017 & -0.001 & 0.036 & 0.058 \\
\hline & Model 2 & 0.007 & -0.016 & 0.029 & 0.554 & 0.004 & -0.011 & 0.019 & 0.578 & 0.016 & 0.001 & 0.031 & 0.038 \\
\hline & Model 3 & 0.015 & -0.024 & 0.055 & 0.449 & -0.030 & -0.065 & 0.005 & 0.090 & 0.034 & 0.009 & 0.058 & 0.007 \\
\hline
\end{tabular}

Table shows associations between number of low $(0.5-1 \mathrm{~g})$, medium $(1-1.5 \mathrm{~g})$ and higher $(>1.5 \mathrm{~g})$ impacts normalised to 7 days, and tibial pQCT measures comprising periosteal circumference (PC), cortical thickness (CT), cortical BMD (BMDc), trabecular density (BMDt) cross sectional moment of inertia (CSMI) and strength strain index (SSI) in 406 participants $(n=401$ for trabecular density). Beta shows SD change in outcome per doubling in number of impacts. Model 1 adjusted for age and error grade, model 2 adjusted for age, error grade, height, fat and lean mass, model 3 as for model 2 plus adjustment for other bands

Table 3: Associations between vertical impacts and DXA measures

\begin{tabular}{|c|c|c|c|c|c|c|c|c|c|c|c|c|c|}
\hline & & \multicolumn{4}{|c|}{ Low impacts } & \multicolumn{4}{|c|}{ Medium impacts } & \multicolumn{4}{|c|}{ Higher impacts } \\
\hline & & Beta & Lower CI & Upper CI & $p$ & Beta & Lower CI & Upper CI & $p$ & Beta & Lower CI & Upper CI & $p$ \\
\hline \multirow[t]{3}{*}{ TH BMD $\left(\mathrm{g} / \mathrm{cm}^{2}\right)$} & Model 1 & -0.071 & -0.134 & -0.008 & 0.027 & -0.034 & -0.080 & 0.012 & 0.147 & 0.001 & -0.048 & 0.050 & 0.963 \\
\hline & Model 2 & 0.043 & -0.021 & 0.108 & 0.188 & 0.015 & -0.028 & 0.059 & 0.492 & 0.020 & -0.025 & 0.065 & 0.383 \\
\hline & Model 3 & 0.087 & -0.030 & 0.203 & 0.145 & -0.055 & -0.158 & 0.047 & 0.287 & 0.030 & -0.042 & 0.101 & 0.417 \\
\hline \multirow[t]{3}{*}{$\mathrm{FN}(\mathrm{g} / \mathrm{cm} 2)$} & Model 1 & -0.059 & -0.122 & 0.003 & 0.063 & -0.036 & -0.082 & 0.009 & 0.119 & -0.006 & -0.055 & 0.042 & 0.798 \\
\hline & Model 2 & 0.012 & -0.055 & 0.078 & 0.731 & -0.007 & -0.052 & 0.039 & 0.773 & 0.005 & -0.042 & 0.051 & 0.843 \\
\hline & Model 3 & 0.072 & -0.048 & 0.192 & 0.237 & -0.072 & -0.177 & 0.033 & 0.180 & 0.033 & -0.041 & 0.107 & 0.380 \\
\hline \multirow[t]{3}{*}{$\mathrm{MNW}(\mathrm{mm})$} & Model 1 & -0.050 & -0.115 & 0.015 & 0.129 & -0.035 & -0.082 & 0.012 & 0.143 & -0.010 & -0.060 & 0.040 & 0.691 \\
\hline & Model 2 & -0.076 & -0.138 & -0.014 & 0.017 & -0.050 & -0.092 & -0.008 & 0.020 & -0.021 & -0.064 & 0.023 & 0.349 \\
\hline & Model 3 & -0.033 & -0.145 & 0.080 & 0.567 & -0.064 & -0.162 & 0.035 & 0.203 & 0.043 & -0.027 & 0.112 & 0.227 \\
\hline \multirow[t]{3}{*}{ CSMI $\left(\mathrm{cm}^{4}\right)$} & Model 1 & -0.090 & -0.152 & -0.027 & 0.005 & -0.060 & -0.105 & -0.014 & 0.010 & -0.015 & -0.064 & 0.033 & 0.534 \\
\hline & Model 2 & -0.069 & -0.128 & -0.009 & 0.024 & -0.053 & -0.093 & -0.013 & 0.010 & -0.015 & -0.056 & 0.027 & 0.489 \\
\hline & Model 3 & 0.008 & -0.099 & 0.115 & 0.882 & -0.108 & -0.202 & -0.014 & 0.025 & 0.067 & 0.001 & 0.133 & 0.045 \\
\hline \multirow[t]{3}{*}{ LS BMD $\left(\mathrm{g} / \mathrm{cm}^{2}\right)$} & Model 1 & -0.161 & -0.227 & -0.095 & 0.000 & -0.091 & -0.139 & -0.043 & 0.000 & -0.032 & -0.084 & 0.019 & 0.220 \\
\hline & Model 2 & -0.059 & -0.127 & 0.010 & 0.096 & -0.040 & -0.087 & 0.006 & 0.091 & -0.007 & -0.055 & 0.041 & 0.783 \\
\hline & Model 3 & -0.012 & -0.137 & 0.113 & 0.851 & -0.082 & -0.191 & 0.028 & 0.143 & 0.064 & -0.014 & 0.141 & 0.107 \\
\hline
\end{tabular}

Table shows associations between number of low $(0.5-1 \mathrm{~g})$, medium $(1-1.5 \mathrm{~g})$ and higher $(>1.5 \mathrm{~g})$ impacts normalised to 7 days, and DXA measures comprising total hip (TH), femoral neck (FN) and lumbar spine (LS) BMD, minimum femoral neck width (MNW) and cross sectional moment of inertia (CSMI), in 408 participants ( $n=401$ for LS BMD). Beta shows SD change in outcome per doubling in number of impacts. Model 1 adjusted for age, model 2 adjusted for age, height, fat and lean mass, model 3 as for model 2 plus adjustment for other bands 
Table 4: Associations between vertical impacts and bone turnover markers

\begin{tabular}{|c|c|c|c|c|c|c|c|c|c|c|c|c|c|}
\hline & & \multicolumn{4}{|c|}{ Low impacts } & \multicolumn{4}{|c|}{ Medium impacts } & \multicolumn{4}{|c|}{ Higher impacts } \\
\hline & & Beta & Lower CI & Upper CI & $p$ & Beta & Lower CI & Upper CI & $p$ & Beta & Lower CI & Upper CI & $p$ \\
\hline \multirow[t]{3}{*}{$\beta-\mathrm{CTX}(\mu \mathrm{g} / \mathrm{L})$} & Model 1 & 0.101 & 0.019 & 0.183 & 0.016 & 0.089 & 0.031 & 0.148 & 0.003 & 0.101 & 0.040 & 0.162 & 0.001 \\
\hline & Model 2 & 0.054 & -0.037 & 0.144 & 0.245 & 0.066 & 0.004 & 0.127 & 0.036 & 0.088 & 0.026 & 0.150 & 0.006 \\
\hline & Model 3 & -0.048 & -0.206 & 0.111 & 0.556 & 0.027 & -0.117 & 0.171 & 0.713 & 0.085 & -0.017 & 0.187 & 0.103 \\
\hline \multirow[t]{3}{*}{$\mathrm{P} 1 \mathrm{NP}(\mu \mathrm{g} / \mathrm{L})$} & Model 1 & 0.049 & -0.028 & 0.126 & 0.214 & 0.056 & 0.000 & 0.111 & 0.048 & 0.095 & 0.038 & 0.152 & 0.001 \\
\hline & Model 2 & 0.036 & -0.050 & 0.122 & 0.412 & 0.048 & -0.011 & 0.106 & 0.110 & 0.088 & 0.029 & 0.147 & 0.003 \\
\hline & Model 3 & -0.021 & -0.172 & 0.129 & 0.780 & -0.039 & -0.174 & 0.097 & 0.577 & 0.127 & 0.031 & 0.224 & 0.010 \\
\hline
\end{tabular}

Table shows associations between number of low $(0.5-1 \mathrm{~g})$, medium $(1-1.5 \mathrm{~g})$ and higher $(>1.5 \mathrm{~g})$ impacts normalised to 7 days, and C-terminal telopeptide ( $\beta$-CTX) and propeptide of type I collagen (P1NP) in 297 participants. Beta shows SD change in outcome per doubling in number of impacts. Model 1 adjusted for age and sample timing (am/pm), model 2 adjusted for age, height, fat and lean mass, model 3 as for model 2 plus adjustment for other bands

exposure to higher vertical impacts in older women is associated with greater lower limb size and strength.

In contrast to higher impacts, low/medium impacts were inversely related to several bone measures. In view of the opposing relationship between low and high impacts and certain bone parameters, and the correlation that exists between impacts in different bands, there was a strong rationale for including adjustment for other bands in our final model. As expected, associations between higher impacts and tibial PC and CSMI were strengthened after adjustment for other impacts. Moreover, a positive association between higher impacts and hip CSMI only became evident after adjustment for low and medium impacts, consistent with the inverse association between low and medium impacts and hip CSMI despite body composition adjustment.

Our observation that higher, as opposed to medium or low, vertical impacts are related to lower limb bone strength is consistent with results of previous interventional studies, as judged by meta-analyses indicating positive effects of high, but not low, impact PA interventions on BMD $[3,18,19]$. In observational studies, equivalent positive relationships between day-to-day participation in high-impact PA and lower limb bone strength has previously been reported in young and middle-aged adults based on PA questionnaires in which different activities are graded according to impact level $[9,10]$, but limited data is available in older populations. Johansson et al. employed actigraph accelerometers to examine relationships between PA and skeletal health in a mixed population aged 70 years, using counts per minute thresholds calibrated according to metabolic rate to define PA intensity; whereas a positive association was observed between moderate PA and tibial PC, no association was observed for vigorous PA [20]. In contrast, previous studies using Newtest accelerometers in which peak accelerations are classified according to $g$ level have yielded similar findings to those reported here in that only high vertical impacts were found to be related to lower limb bone outcomes in adolescents and premenopausal women $[13,14,21]$.

Individuals whose higher impact counts were in the top quartile (i.e. greater than 106 counts per weeks) experienced approximately fourfold more high impacts compared with those in the bottom quartile (i.e. less than 17 counts per week), which translates into a 0.13 SD greater hip CSMI. Since greater hip CSMI is an established protective factor for hip fracture [22], this association between higher impacts and CSMI is expected to lead to a reduced risk of hip fracture. However, no association was observed with hip BMD, which is more strongly related to hip fracture risk compared with hip CSMI [22]. Therefore, although our findings support our prior hypothesis that positive relationships between day-to-day PA and bone strength in older women are explained by exposure to higher impacts, any benefit in terms of fracture prevention is likely to be limited.

A possible explanation for this limited association is that the quartile of our study population with the greatest number of high impacts only experienced approximately 15 vertical impacts $>1.5 \mathrm{~g}$ daily. In terms of the activities responsible for high impacts, $1.5 \mathrm{~g}$ exceeds that associated with walking (typically $0.5-1.0 \mathrm{~g}$ ), but is achieved in the majority of aerobics class exercises undertaken by older individuals, particularly those with a jumping component [23]. Aerobics exercise participation was one of the commonest forms of high-impact PA in COSHIBA as assessed by questionnaire, and presumably contributed to the positive relationship we observed between impacts $>1.5 \mathrm{~g}$ and lower limb bone strength [24]. Alternatively, the weak associations we observed could be a reflection of the relatively low levels of impact involved, and it may be that stronger impacts, closer to the 4-g osteogenic threshold identified in studies of younger individuals, are required to produce a significant benefit in terms of bone strength and fracture risk. Though impacts of this level are rarely achieved in unselected individuals, in our previous exercise study, we found that impacts within this range are 
readily achievable with only minor modifications to the class protocol [23].

Our fully adjusted model suggested that higher, but not medium or low, impacts exert a net positive effect on bone formation, as reflected by a positive association with P1NP but not $\beta$-CTX. Although we speculated that this might represent a mechanism by which higher impacts improve bone strength, these associations were found to be unaffected by P1NP adjustment. Few previous observational studies have examined relationships between day-to-day levels of PA and bone markers. Our results are potentially consistent with a previous study of 530 premenopausal women, in which weight-bearing PA, as assessed by questionnaire, was found to be positively related to P1NP levels, whereas no association was observed with $\beta$-CTX; however, these relationships were not broken down according to PA intensity or type [25].

Some of the inverse associations we observed between low/medium impacts and bone measures were attenuated after adjustment for body composition, presumably reflecting the fact that PA is known to be inversely related to fat mass, of which the latter is positively related to BMD involving a causal pathway between fat and bone [26]. Interventional studies provide further evidence that weight loss including lowintensity exercise has a net adverse effect on BMD [27]. However, several of these low/medium impact inverse associations, e.g. with MNW and tibial CSMI, persisted following adjustment for body composition. Whereas older individuals participating in more low and moderate impact PA may have had a lower fat mass over their life course, leading to longterm effects on their skeleton, this may be poorly reflected by body composition measurements taken at a mean of 77 years of age. Alternatively, it may be that low or medium impacts exert hitherto unrecognised effects on bone metabolism. For example, in our minimally adjusted analyses, low impacts were positively associated with the bone resorption marker $\beta$-CTX, whereas no association was observed with the formation marker P1NP, suggesting a net balance of resorption over formation leading to bone loss.

\section{Limitations}

There are several inherent limitations in use of accelerometry to measure day-to-day PA. For example, an individual's daily activity may be altered as a result of being recorded. In addition, PA levels are likely to be affected by seasonal influences. However, seasonal variation in weather patterns in the southwest of the UK is limited, and any effect on PA levels would have reduced the strength of associations by increasing variability, rather than leading to spurious associations through introducing bias. In terms of other limitations, our crosssectional study design limits the ability to infer causality, and we are unable to distinguish whether the association we observed between higher impacts and bone strength reflects an osteogenic effect of high-impact PA on bone, or arises from confounding. That said, our results were unaffected by adjustment for a range of confounders ascertained by contemporaneous questionnaire. Another important limitation is that given the relatively small effect sizes observed, and the multiple outcomes and models examined, we are unable to exclude the possibility of a type I error. A further limitation is the generalisability of our results to the wider population. Although our study was based on a population-based cohort, $44 \%$ of those invited attended the research clinic, which is likely to have resulted in selection of a relatively healthy subgroup, consistent with which those attending were approximately 4 years younger than those who did not attend.

\section{Conclusions}

We investigated relationships between vertical acceleration peaks as measured by 7-day accelerometer recordings and lower limb skeletal strength, in a population-based cohort of older women. Despite their rarity and the relatively low $1.5 \mathrm{~g}$ threshold, day-to-day levels of higher vertical impacts were positively associated with lower limb bone size and strength, whereas equivalent associations were not seen for medium or low impacts. These findings are consistent with the possibility that despite the low levels of impacts achieved, day-to-day PA in older women is beneficial for their skeletal health.

Acknowledgements We are extremely grateful to all the study participants who took part in this study, the fieldworkers, laboratory technicians and research scientists. This study was funded by the Medical Research Council, grant ref. MR/K024973/1.

\section{Compliance with ethical standards}

Conflicts of interest None.

Open Access This article is distributed under the terms of the Creative Commons Attribution 4.0 International License (http:// creativecommons.org/licenses/by/4.0/), which permits use, duplication, adaptation, distribution and reproduction in any medium or format, as long as appropriate credit is given to the original author(s) and the source, a link is provided to the Creative Commons license and any changes made are indicated.

\section{References}

1. Tan VP, Macdonald HM, Kim S, Nettlefold L, Gabel L, Ashe MC, McKay HA (2014) Influence of physical activity on bone strength in children and adolescents: a systematic review and narrative synthesis. J Bone Miner Res 29:2161-2181

2. Babatunde OO, Forsyth JJ, Gidlow CJ (2012) A meta-analysis of brief high-impact exercises for enhancing bone health in premenopausal women. Osteoporos Int 23:109-119 
3. Martyn-St James M, Carroll S (2009) A meta-analysis of impact exercise on postmenopausal bone loss: the case for mixed loading exercise programmes. Br J Sports Med 43:898-908

4. Allison SJ, Folland JP, Rennie WJ, Summers GD, Brooke-Wavell K (2013) High impact exercise increased femoral neck bone mineral density in older men: a randomised unilateral intervention. Bone 53:321-328

5. Rubin LT, Lanyon CE (1984) Regulation of bone formation by applied dynamic loads. J Bone and Joint Surg 66A:397-402

6. Shah K, Armamento-Villareal R, Parimi N, Chode S, Sinacore DR, Hilton TN, Napoli N, Qualls C, Villareal DT (2011) Exercise training in obese older adults prevents increase in bone turnover and attenuates decrease in hip bone mineral density induced by weight loss despite decline in bone-active hormones. J Bone Miner Res 26 : 2851-2859

7. Scott JP, Sale C, Greeves JP, Casey A, Dutton J, Fraser WD (2012) Effect of fasting versus feeding on the bone metabolic response to running. Bone 51:990-999

8. Maimoun L, Sultan C (2011) Effects of physical activity on bone remodeling. Metabolism 60:373-388

9. Mori T, Ishii S, Greendale GA, Cauley JA, Sternfeld B, Crandall CJ, Han W, Karlamangla AS (2014) Physical activity as determinant of femoral neck strength relative to load in adult women: findings from the hip strength across the menopause transition study. Osteoporos Int 25:265-272

10. Neville CE, Murray LJ, Boreham CA, Gallagher AM, Twisk J, Robson PJ, Savage JM, Kemper HC, Ralston SH, Davey Smith G (2002) Relationship between physical activity and bone mineral status in young adults: the Northern Ireland young hearts project. Bone 30:792-798

11. Heikkinen R, Vihriala E, Vainionpaa A, Korpelainen R, Jamsa T (2007) Acceleration slope of exercise-induced impacts is a determinant of changes in bone density. J Biomech 40:2967-2974

12. Vainionpaa A, Korpelainen R, Leppaluoto J, Jamsa T (2005) Effects of high-impact exercise on bone mineral density: a randomized controlled trial in premenopausal women. Osteoporos Int 16: 191-197

13. Deere K, Sayers A, Rittweger J, Tobias J (2012) Habitual levels of high, but not moderate or low, impact activity are positively related to hip BMD and geometry: results from a population-based study of adolescents. J Bone Miner Res 27(9):1887-1895

14. Deere K, Sayers A, Rittweger J, Tobias JH (2012) A cross-sectional study of the relationship between cortical bone and high-impact activity in young adult males and females. J Clin Endocrinol Metab 97(10):3734-3743

15. Deere KC, Hannam K, Coulson J et al (2016) Quantifying habitual levels of physical activity according to impact in older people: accelerometry protocol for the VIBE study. Journal of Ageing and Physical Activity 24:290-295

16. Clark EM, Gould V, Morrison L, Ades A, Dieppe P, Tobias JH (2011) Randomised controlled trial of a primary care-based screening programme to identify older women with prevalent osteoporotic vertebral fractures (COSHIBA). J Bone Miner Res 27(3):664-671

17. Clark EM, Gould VC, Morrison L, Masud T, Tobias J (2012) Determinants of fracture risk in a UK-population-based cohort of older women: a cross-sectional analysis of the cohort for skeletal health in Bristol and Avon (COSHIBA). Age Ageing 41:46-52

18. Martyn-St James M, Carroll S (2008) Meta-analysis of walking for preservation of bone mineral density in postmenopausal women. Bone 43:521-531

19. Martyn-St James M, Carroll S (2010) Effects of different impact exercise modalities on bone mineral density in premenopausal women: a meta-analysis. J Bone Miner Metab 28:251-267

20. Johansson J, Nordstrom A, Nordstrom P (2015) Objectively measured physical activity is associated with parameters of bone in 70 year-old men and women. Bone 81:72-79

21. Vainionpaa A, Korpelainen R, Vihriala E, Rinta-Paavola A, Leppaluoto J, Jamsa T (2006) Intensity of exercise is associated with bone density change in premenopausal women. Osteoporos Int 17:455-463

22. Kaptoge S, Beck TJ, Reeve J, Stone KL, Hillier TA, Cauley JA, Cummings SR (2008) Prediction of incident hip fracture risk by femur geometry variables measured by hip structural analysis in the study of osteoporotic fractures. J Bone Miner Res 23:18921904

23. Hannam K, Deere KC, Worrall S, Hartley A, Tobias JH (2016) Characterisation of vertical accelerations experienced by older people attending an aerobics class designed to produce high impacts. Journal of ageing and physical activity 24:268-274

24. Hannam K, Deere KC, Hartley A, et al. (2016) A novel accelerometer-based method to describe day-to-day exposure to potentially osteogenic vertical impacts in older adults: findings from a multi-cohort study. Osteoporos Int

25. Adami S, Gatti D, Viapiana O, Fiore CE, Nuti R, Luisetto G, Ponte M, Rossini M, Group BS (2008) Physical activity and bone turnover markers: a cross-sectional and a longitudinal study. Calcif Tissue Int 83:388-392

26. Kemp JP, Sayers A, Davey-Smith G, Tobias JH, Evans DM (2016) Using Mendelian randomization to investigate a possible causal relationship between adiposity and increased bone mineral density at different skeletal sites in children. Int J Epidemiol In Press

27. Soltani S, Hunter GR, Kazemi A, Shab-Bidar S (2016) The effects of weight loss approaches on bone mineral desnity in adults: a systematic review and meta-analysis of randomised control trials. Osteoporosis International In Press 